Revue de l'Institut des langues et cultures

d'Europe, Amérique, Afrique, Asie et Australie

23 | 2015

Le national-socialisme dans son cinéma

\title{
Une analyse de Heimkehr (G. Ucicky, 1941)
}

Gustav Ucickys Film Heimkehr (1941): eine Analyse

\section{Éric Dufour}

\section{OpenEdition}

Journals

Édition électronique

URL : http://journals.openedition.org/ilcea/3393

DOI : 10.4000/ilcea.3393

ISSN : 2101-0609

Éditeur

UGA Éditions/Université Grenoble Alpes

Édition imprimée

ISBN : 978-2-84310-305-6

ISSN : $1639-6073$

\section{Référence électronique}

Éric Dufour, "Une analyse de Heimkehr (G. Ucicky, 1941)», ILCEA [En ligne], 23 | 2015, mis en ligne le 09 juillet 2015, consulté le 19 avril 2019. URL : http://journals.openedition.org/ilcea/3393 ; DOI :

10.4000/ilcea.3393

Ce document a été généré automatiquement le 19 avril 2019

(c) ILCEA 


\title{
Une analyse de Heimkehr (G. Ucicky, 1941)
}

Gustav Ucickys Film Heimkehr (1941): eine Analyse

\author{
Éric Dufour
}

1 Heimkehr (Retour, exploité en France pendant la guerre sous le titre Retour au pays) est un film de fiction, au contenu de propagande explicite, en noir et blanc, réalisé par Gustav Ucicky et écrit par Gerhard Menzel, qui date de 1941. Il a reçu, lorsqu'il est sorti, le prédicat suprême, «Film de la nation » (Film der Nation). Il fait partie des quarante films environ (Capurro \& Grimm, 2004: 83; Krah, 2000: 19) interdits par les Alliés après la guerre (Vorbehaltsfilme), à cause de leur contenu politique douteux, qui ne peuvent être projetés que sous certaines conditions, à savoir un contexte explicatif scientifique qui peut dynamiter l'effet que le film peut produire sur un public non prévenu et naïf.

2 Je ne dis rien des conditions de production du film, ni de sa réception, dans la mesure où ces deux points sont amplement développés par Gerald Trimmel dans le livre qu'il a consacré au film, Heimkehr, Strategien eines nationalsozialistischen Films, publié en 1998, et qui a par ailleurs écrit sur ce film deux articles qu'on trouve sur internet et qui résument sa pensée ${ }^{1}$. Malgré l'implication de Goebbels lui-même dans le projet (Fox, $2000: 38$ ) et, conséquemment, les critiques élogieuses, ou plutôt les Kunstbetrachtungen (Helm, 2006 : 6) que le film reçoit quand il sort, il est un relatif échec public. Reste que Heimkehr, en 1944, était un des 6 films préférés des jeunes (Sander, 1944 : 118-119).

Heimkehr est un film tourné pour justifier l'invasion de la Pologne en septembre 1939. Remarquons d'abord que c'est une justification tardive, et ensuite que, comme c'est le cas pour toutes les autres actions de propagande cinématographique, il s'agit pour les nazis de saturer l'espace public, car, outre les journaux et la radio, ils doublent les films de fiction avec des films documentaires (Kulturfilm, Dokumentar, mais aussi la Wochauschau) qui véhiculent le même message, dans un cas en prétendant donner au spectateur qui reste extérieur une pure information objective, dans l'autre cas en l'impliquant, affectivement, dans une histoire où l'arrière-fond retrouve et utilise les mêmes éléments narratifs et esthétiques que ceux qu'on trouve dans le documentaire (Fox, 2007 : 30-47; 
Rentschler, $2002: 20$ ). En ce qui concerne l'invasion de la Pologne, Feuertaufe et Feldzug in Polen sont, dès 1939-1940, les pendants documentaires de Kampfgeschwader Lützow puis de Heimkehr. Ils entretiennent le même rapport que, par exemple, Der ewige Jude, d'un côté, et, de l'autre, Jud Süss ou Die Rothschilds. La complémentarité des films documentaires ou des actualités et des films de fiction est, en outre, soutenue par la grande unité qui se tisse dans l'espace fictionnel cinématographique nazi où, de Der Hitlerjunge Quex et Flüchtlinge jusqu'à Kolberg, on retrouve les mêmes piliers fondateurs de ce cinéma de propagande nazi.

4 De la ligne ouverte par Quex, on retrouve, face aux Allemands impassibles, raisonnables, prudents et non violents, qui simplement se défendent quand ils sont attaqués, l'ennemi sans visage, fou et furieux, plein de haine, qui attaque, attaque et attaque encore, sans raison. Ce qui répond dans ce film à la scène où, dans Quex, les Hitlerjungen partent tranquillement à la campagne faire un petit feu de camp sympa en chantant tranquillement sans embêter personne, agressés à la gare par les méchants communistes, c'est la grande impassibilité, quasi stoïcienne, des Allemands au début de Heimkehr, lorsque les Polonais brûlent leur école, crient et courent dans tous les sens. Pour ce qui est de Flüchtlinge, Heimkehr s'inscrit dans ce genre appelé le Heims-in-Reich Film qui fleurit à partir de 1933 (il faut aussi nommer Ein Mann will nach Deutschland la même année que Flüchtlinge). En ce qui concerne Kolberg, le film présente, encore une fois (mais c'est la dernière), une expression du Führerprinzip dans la personne de Gneisenau : il suffit qu'il parle, et il a ce petit quelque chose de plus qui fait qu'il fédère, qu'on lui obéit, parce que, quand il parle, c'est le peuple qui parle à travers lui. Il est le peuple allemand.

Heimkehr, surtout, retrouve cette constante structurelle du cinéma de fiction mis en place par la propagande nazi, dont Quex et Kolberg sont les deux termes extrêmes. C'est le cinéma comme performatif, c'est-à-dire le cinéma qui instaure une réalité, la réalité vraie, non déformée, qu'il faut savoir voir lorsqu'on passe derrière les apparences. C'est l'utilisation nazie du cinéma: le cinéma comme pouvoir pour instaurer la vérité, pour rétablir une vision juste du monde. La réalité n'est plus quelque chose qui préexiste au cinéma, et sur quoi il devrait se régler, comme si elle avait une transcendance : chez les nazis, c'est le cinéma, c'est l'image qui engendre la réalité et la donne sans tromperie, sans mensonge.

Dans Heimkehr, l'invasion de la Pologne est justifiée par la violence polonaise vis-à-vis des Allemands. Tout le film fait fond sur cette idée, et la développe comme on développe un thème musical.

7 Une fois cette unité fondamentale mais complètement générale soulignée, il faut insister, à propos de Heimkehr, sur les paradoxes et les contradictions qu'on y trouve, au niveau du contenu très précis du film, c'est-à-dire relativement à ce qu'on nomme l'idéologie nazie. Et l'on verra que, d'un certain point de vue, ces contradictions sont très cohérentes. Ce sera l'objet de mon intervention. Alors que le centre de cette intervention porte sur le contenu du film, c'est-à-dire et plus exactement sur le texte politique et idéologique mis en œuvre par l'histoire, la conclusion portera sur la forme, c'est-à-dire sur le récit - et il soulignera les stratégies narratives et cinématographiques mises en œuvre par Heimkehr, afin d'ouvrir sur l'après Heimkehr, c'est-à-dire sur ce qui peut légitimement être mis en œuvre, du point de vue de l'éthique cinématographique, quand, aujourd'hui, on dénonce la barbarie nazie.

8 Trimmel, dans les nombreux textes qu'il a consacrés à Heimkehr, parle sans cesse de la figuration que ce film donne au sous-homme, à l'Untermensch. Il souligne que la sous- 
humanité, face à l'Allemand, est représentée comme pure bestialité et violence, citant des textes d'idéologues nazis sur ce sujet (Trimmel: «Der nationalsozialistische Spielfilm „Heimkehr“. Strategien der Manipulation und Propaganda »). Les textes sont certes intéressants, mais plaqués de l'extérieur sur le film et témoignant, pour cette raison, d'une totale violence herméneutique. La première violence herméneutique de Trimmel, qui n'est pas la plus importante, consiste à affirmer que le sous-homme est doublement représenté dans le film : il y a les Polonais, mais ceux-ci ne sont selon Trimmel que des exécutants (Täter) d'une violence dont les auteurs (Urheber) sont en fait les juifs, caché derrière ${ }^{2}$. Et c'est parce que le juif est caché derrière qu'il est quasiment absent du film, écrit Trimmel. Mais c'est justement parce qu'il est caché, donc absent, comme le reconnaît Trimmel, si l'on excepte d'abord le jeune juif au début, très actif dans la mise à sac de l'école, puis le marchand juif, dont on ne saurait supposer qu'il est la cause véritable de toutes les exactions pratiquées par les Polonais. Donc, dans le règne de la violence qui s'exerce contre les Allemands, il y a, outre les Polonais, une présence des juifs ${ }^{3}$. Mais ce n'est pas tout. Après le massacre de l'école, la deuxième scène importante du film, son deuxième climax, c'est la séance de cinéma, où Marie Thomas, l'héroïne, son mari et un ami se font conspuer par les Polonais, qui se divise en trois moments : dans la salle de cinéma, puis dans le hall où Marie assiste son mari blessé, et enfin à l'hôpital où elle le mène pour qu'il y soit soigné. Il est remarquable que, dans le deuxième moment, lorsque l'héroïne assiste son mari, couché dans le hall, et discute avec le propriétaire ou gérant des lieux qui l'invite proprement à quitter les lieux, alors que son mari ne peut plus bouger, on voit clairement, en arrière-plan, des affiches de films américains (on lit nettement le logo MGM, le nom de John Barrymore, etc., ce qui renvoie d'ailleurs au début de la séquence, donc au premier moment où le montage en $\mathrm{L}$ nous fait passer de l'extérieur de la salle à l'intérieur au moyen du logo sonore de la 20th Century Fox). Voilà quelque chose que Trimmel ne remarque pas, et c'est bien dommage. On est en 1941, c'est-à-dire l'année où les films américains sont interdits en Allemagne. L'insistance du film sur la présence des Américains en Pologne, et le fait que cette présence soit signalée en douce, ici à travers son industrie cinématographique, visent le même effet que la présence de juifs parmi les Polonais : non pas assigner une cause au mal, mais montrer la grande collaboration des ennemis de l'Allemagne, Polonais, juifs et Américains - telle une menace inassignable, comme dans un film d'horreur (L'invasion des profanateurs, The Thing de Carpenter ${ }^{4}$ ), d'autant plus menaçante qu'elle peut prendre tous les visages. La menace, tel le Dieu de la théologie négative, ne peut être définie que par ce qu'elle n'est pas : la menace, c'est ce qui n'est pas allemand.

9 La deuxième violence herméneutique de Trimmel est plus importante, parce qu'elle met en jeu quelque chose de fondamental.

Ce qui est remarquable, dans le film, c'est qu'il n'est jamais question de sous-homme ou de sous-humanité. Mais il y est en revanche question d'autre chose - et c'est ça qui est remarquable. Dans le troisième moment de la séance de cinéma, donc à l'hôpital, le secrétaire de l'hôpital refuse malgré l'insistance de l'héroïne qu'on soigne son mari. Il souligne qu'on n'y soigne que les Polonais, pas les étrangers, et parle des gens (Leuten) d'une manière vague et abstraite. Et l'héroïne, Marie, de s'énerver en disant : les gens, les gens ! Mais c'est d'une vie humaine qu'il s'agit. Elle en appelle à Dieu, traite le secrétaire d'inhumain (Unmensch) et, lorsque la caméra revient sur elle (plan rapproché) après le contrechamp sur la croix qu'elle montre du doigt, elle dit : n'est-ce là qu'une décoration pour vous? 
11 Ce n'est pas le seul film nazi qui en appelle à l'humanité, promue valeur positive, c'est-àdire qui fait valoir l'humanisme. Erbkrank, un Kulturfilm de 1936, en appelait à la protection des handicapés qui sont des êtres humains. Et, de même, Hans Westmar, qui fait partie de la trilogie relevant de l'offensive contre le communisme en 1933, faisait valoir que les communistes sont des gens qui n'hésitent pas à tuer un jeune homme sans respect aucun de la dignité humaine. Voilà le grand paradoxe qu'on ne note jamais, parce qu'on lit les films en fonction de l'hypothèse d'une idéologie nazie qu'on plaque dessus, sans prêter attention à ce qu'ils contiennent effectivement.

12 Cet élément n'est pas le seul qui contrevient à ce qu'on nomme l'idéologie nazie. Il y a aussi, dans ce film, le rôle et la place de la femme. Lorsque, dans le troisième moment fort du film, qui est vraiment son apothéose, à savoir l'enfermement de tous les membres de la communauté allemande dans des geôles, prélude à leur exécution imminente, les Allemands commencent à craquer, le film révèle le véritable statut d'héroïne de Marie Thomas. C'est le moment où, face à un homme, pourtant d'apparence virile, qui craque, elle lui intime l'ordre de se calmer, et se lance dans un long monologue, très célèbre et cité dans de nombreux livres, sur le Heimat et le Heimkehr. Marie Thomas est une femme qui a des couilles, on l'avait compris depuis le début (et Trimmel souligne d'ailleurs que le film gomme toute érotisation du personnage). Mais, plus encore, c'est elle qui incarne la force et la valeur suprême du nazisme, que résume l'expression de Führerprinzip. Telle l'unité catégorielle dans la Critique de la raison pure de Kant, elle ordonne la diversité et la soude autour des mots "allemand », " Allemagne ", déclinés et répétés à l'infini. Toute individualité disparaît, au point que la mort de l'individu n'est rien, puisque c'est la cessation de vie d'une partie de l'Allemagne pour que cette grande Allemagne vive. Voilà qui semble aller contre l'idéologie nazie dans sa prétendue cohérence. À nouveau, Trimmel botte en touche, écrivant que le triomphe de la femme sur l'homme est tout apparent ${ }^{5}$. Or, non seulement il n'est pas apparent, puisqu'il est la vérité du film, son achèvement suprême préparé par tout ce qu'on a vu avant, mais il faut également souligner que, là encore, c'est-à-dire sur les femmes, on trouve tout et son contraire chez les nazis. Pierre Ayçoberry souligne qu'il existe chez les nazis un « double discours sur les femmes » (1998: 193-195), et, pour ce qui est de la femme au cinéma, Antje Ascheid insiste sur le fait qu'on trouve, dans le cinéma nazi, "un certain nombre de discours féministes » (2003 : 215) qui s'harmonisent peu avec l'idéal nazi de la femme.

13 Ces paradoxes se retrouvent dans le concept de Heimat. Dans Heimkehr, le Heimat, c'est d'abord le sang, l'hérédité, c'est-à-dire le sol qui en est la métaphore. En témoigne d'abord le générique du film : le mouvement descendant de la caméra, au début, sur la terre avant que les caractères s'inscrivent, désigne le sens du Heimkehr et donc du Heimat, précède le mouvement ascendant, à la fin du générique, qui rejoint le ciel et lui accorde à nouveau les 2/3 de l'image, suivi par les deux intertitres qui donnent le sens de l'histoire qu'on va voir. En témoigne ensuite le monologue de Maria, où ce qui est allemand, ce n'est pas seulement la langue, mais le moindre brin d'herbe et tous les animaux. Mais en même temps, d'un autre côté, l'Allemagne, c'est la culture : c'est l'école qu'on brûle au début (tout un symbole), et puis la musique, celle qu'on chante et qui nous réunit, par laquelle on se fond en une unité où se dissout toute individualité. À la fin de la séquence de la geôle, Marie dit : " chantons, et chantons notre chanson » (il s'agit évidemment de Nach der Heimat möcht'ich wieder) ${ }^{6}$.

14 Ce paradoxe se retrouve dans le traitement des animaux. 
Dans Kampfgeschwader Lützow, un soldat allemand sauve un chien polonais, donc ennemi. Mais, après tout et pour reprendre la formule attribuée à Stéphanie de Monaco, les animaux ne sont-ils pas des êtres humains comme les autres? Il s'agit donc en somme d'un geste humaniste. Ce geste va dans le sens d'une certaine image, celle que les nazis voulaient donner d'eux-mêmes, l'image selon laquelle on protège les animaux et on leur confère des droits. Mais outre que cette image ne correspond pas à la réalité, comme l'a montré Élisabeth Hardouin-Fugier (2002 : 129-151), en établissant que le thème du droit des animaux n'est qu'un instrument de propagande qui masque, dans les faits, l'assimilation de l'animal à une marchandise, il y a autre chose qui, pour notre propos est intéressant et apparaît dans notre film. À côté des valeurs humanistes qui affirment l'égalité des hommes, et qui sont explicitement utilisées dans les films que j'ai cités, on trouve aussi dans de nombreux films et d'une manière explicite, contre tout humanisme, un racisme évident. La thèse exemplifiée par Kampfgeschwader Lützow, qui représente l'équivalent, pour l'animal, de l'humanisme (les chiens polonais sont des chiens comme les autres, donc d'abord chien, et accessoirement polonais), coexiste donc avec une thèse, apparaissant dans d'autres films, qui est l'équivalent du racisme appliqué aux animaux. Dans Heimkehr, la séquence où Marie, au début, se rend chez le maire, pour contester la destruction de l'école, commence par un très remarquable travelling qui, comme toujours chez Ucicky (j'y reviendrai), lie plusieurs mouvements, et part du chien du maire, pour aller sur le maire, puis sur Marie. Le chien grogne et on voit qu'il n'aime pas Marie, exactement comme son maître. Autrement dit, avant d'être un chien, ce chien est d'abord polonais, donc mauvais et violent. Le chien n'est pas le seul à être d'abord polonais avant d'être chien. L'oiseau sauvé par les Allemands lors de la destruction de l'école est d'abord allemand avant d'être oiseau : j'en veux pour preuve le monologue de Marie, où la chose est explicitement dite. Dans ... reitet für Deutschland, le cheval, immense, sublime pur sang, est allemand - son physique et son caractère, pendant tout le film, l'indiquent : et il subit comme son maître la dégradation de l'Allemagne et son humiliation pendant cette horrible époque que fut l'Allemagne de Weimar, avant sa renaissance grâce au Reich millénaire. Il ne faut pas oublier qu'un an après Kampfgeschwader Lützow une loi interdit aux juifs d'avoir des animaux domestiques et a pour conséquence qu'on enlève et qu'on tue les animaux considérés « perdus pour l'espèce (artvergessen)» (Klemperer, 1996 : 140), concept qui fournit donc l'équivalent, pour les animaux, de ce qu'est le racisme pour les hommes : tel fut le sort de Muschel, le chat de Viktor Klemperer.

Les historiens du nazisme, tel Norbert Frei aujourd'hui, ont insisté sur le caractère contradictoire de l'idéologie nazie (Frei, $1994: 167,190)$. Mais il ne suffit pas de dire ça. Mon hypothèse est exactement celle que Max Horkheimer fait à propos de l'idéologie mussolinienne, dans l'article de 1937 "La dernière attaque contre la métaphysique " (Horkheimer, 1978 : 242-243). Pour l'adapter à la spécificité des nazis, je dirai que, s'il y a bien des contre-valeurs, donc des valeurs réactives (le juif, le sous-homme, etc.), il n'y a pas de valeur positive, d'idéologie positive. Il n'y a qu'un but : la guerre et l'accroissement de la puissance. Et, relativement à cet unique but, totalement vide, l'idéologie, au sens positif du terme, varie selon l'ennemi qu'il convient de mettre en avant à un moment donné et dans une situation donnée ${ }^{7}$. Bref, tout est bon dans une situation donnée pour abattre un ennemi déterminé, raison pour laquelle les nazis font feu de tout bois, et font valoir, par exemple dans Heimkehr (ou déjà Hans Westmar ou Erbkrank), un humanisme dont on sait que, par ailleurs, donc en soi, ils n'en soutiennent pas les valeurs. De même 
pour la femme, de même pour les animaux, etc. Et c'est en ce sens là (et uniquement en ce sens là) que l'idéologie nazie est très cohérente ${ }^{8}$.

17 Je passe désormais, en guise de conclusion, au récit c'est-à-dire à la forme proprement cinématographique. C'est par l'excellence de cette forme que j'explique le succès du film auprès des jeunes. Il faut distinguer deux éléments qui collaborent à cette forme, représentés par le duo Menzel-Ucicky, déjà auteurs de Flüchtlinge. Le premier c'est donc le scénario, habilement dosé : comme dans une ouverture de Rossini, les déterminations s'ajoutent les unes aux autres dans une progression très bien gérée dont le climax, je l'ai dit, est le grand monologue de Marie. D'abord on détruit l'école - donc l'objectivation de la culture allemande. Puis ce sont les hommes qu'on détruit avec un nouveau crescendo : le mari de l'héroïne blessé à mort, le père de l'héroïne qui devient aveugle après être attaqué, la femme qui est lapidée, puis la population allemande toute entière. Cela jusqu'au dénouement in extremis : l'invasion de la Pologne qui sauve les Allemands - et l'histoire entre dans l'Histoire avec, à la frontière, ce portrait d'Hitler sur lequel s'achève le film. Je ne comprends pas comment Trimmel peut reprocher au film son aspect bavard et, surtout, son aspect grossier du point de vue narratif ${ }^{9}$. De nombreux historiens du cinéma nazi ont souligné que l'ambition du cinéma nazi était d'égaler son modèle, à savoir le cinéma hollywoodien (Brockmann, $2010: 12,139)$. L'aspect grossier du scénario n'est rien d'autre que cette schématisation propre au récit hollywoodien, qui fait qu'on élague et qu'on ne conserve, comme dit Truffaut commentant Hitchcock, que ce qui fait avancer l'action et possède un sens dans l'économie de la narration. Quant à l'aspect bavard, qui lui aussi est une dimension d'un certain cinéma hollywoodien, il ne contrevient nullement à la qualité cinématographique quand, justement, il est magnifié par la mise en scène, ce qui est proprement le cas ici.

Car il faut en venir à Ucicky. Immense Ucicky ${ }^{10}$. Ucicky a d'abord été le chef opérateur de Michael Kertesz, ensuite rebaptisé aux États-Unis Michael Curtiz, un des plus grands réalisateurs hollywoodiens dans les films duquel, justement, aucun plan n'est gratuit : de même que chaque élément du scénario a toujours une fonction dans la progression du récit, tout plan prend un sens dans l'économie de la totalité et possède une utilité dans la narration. Heimkehr relève de la même manière de concevoir le cinéma - c'est-à-dire qui respecte les règles qui sont celles de ce qu'on appelle le cinéma classique hollywoodien. Voilà le premier point. Mais il y en a un second. Comme en littérature, il y a un style reconnaissable de tout grand cinéaste, un peu comme une marque de fabrique, par exemple la petite phrase proustienne. Dans les films de Veit Harlan, il y a aussi un style, reconnaissable par exemple dans ces petits travellings immersifs systématiques qui renforcent l'incorporation du spectateur dans l'action. Il y a peu de cinéastes à proprement parler dans l'Allemagne nazie. Cependant, Ucicky, justement, en est un. Ucicky a une manière bien à lui d'organiser la séquence, donc de filmer les situations et les dialogues que Trimmel qualifie de bavards : non pas un plan d'ensemble d'abord, puis des plans serrés qui découpent des élément de l'ensemble. Mais, au contraire, Ucicky montre d'abord les parties, puis l'ensemble. Et cela, non pas au moyen de plans fixes qui s'ajouteraient mécaniquement les uns aux autres, mais au moyen de travellings latéraux ou arrière, toutefois jamais rectilignes, mais toujours en arc de cercle, témoignant d'une grande élégance et d'une grande habileté dans la construction de l'espace. C'est le style Ucicky. Le fait que Trimmel, dans «Der nationalsozialistische Spielfilm „Heimkehr“. Strategien der Manipulation und Propaganda ", consacre toute une partie à analyser l'échelle des plans dans Heimkehr, témoigne à mon sens d'une double méprise. La 
première est due au fait que l'échelle varie dans la séquence, à cause du mouvement des personnages et de celui de la caméra. La seconde tient au fait que Ucicky, et donc Heimkehr, c'est le mouvement systématique de la caméra. Si l'on veut faire une analyse de Heimkehr, c'est alors de ces mouvements qu'il faut parler: travellings et/ou panoramiques, accompagnateurs ou bien descriptifs, lents ou rapides, ou bien même filés par moments (soit pour souligner un effet de surprise ou de violence, soit pour s'aligner sur la narration). On voit bien qu'Ucicky préfère le plan séquence au montage des différents plans, et tente, autant qu'il le peut, d'établir une continuité : exemplaire à cet égard est le très long travelling lorsque les Allemands écoutent en cachette la radio allemande, avant de se faire prendre par les Polonais (où il n'y a qu'une seule coupure, à la fin de la séquence, sur le père de l'héroïne devenu aveugle, qui annonce précisément l'irruption des Polonais dans le raccord suivant). Sur ce point, Ucicky est le digne successeur des représentants de la entfesselte Kamera qui apparaît dans le cinéma allemand des années vingt, d'une part, et, d'autre part, cette organisation de la séquence témoigne toujours d'un grand sens de l'économie dans le choix du point de vue : il faut savoir choisir le point de vue à partir duquel on pourra passer continument à ce qui suit (quelqu'un qui entre, ou quelqu'un qui sort, ou quelqu'un qui bouge dont on accompagne le mouvement sans jamais avoir besoin de changer de point de vue).

Mais ce talent proprement cinématographique ne va pas sans sa contrepartie, à savoir ce au service de quoi il est, et qui rend le film d'autant plus abject. Conformément à la stratégie qui surgit avec Quex, le cinéma nazi opère un renversement systématique de la réalité - c'est sa fonction performative. Ici, ce sont les Allemands qui sont menacés, qui écoutent la radio en secret, et qui, à la fin, vont être massivement exterminés par les Polonais. Le cinéma nazi donne pour la première fois au cinéma une image de l'extermination de masse au moment même où les nazis la mettent en œuvre. C'est cela qui est proprement scandaleux et qui, à mes yeux, fait que le degré d'abjection de Heimkehr est peut-être le plus grand de tous les films nazis. Cette extermination de masse (ou du moins sa préparation) est montrée très concrètement, et avec tout le talent d'Ucicky. Heimkehr, du début jusqu'à la fin, est construit sur un seul principe : l'insistance sur les conditions très concrètes d'avilissement, d'humiliation et de négation de la dignité humaine, subies par des êtres humains innocents, dont le seul souhait est de rentrer chez eux, et qui sont ravalés au rang de bêtes - mieux encore : qui sont même traités d'une manière pire que des bêtes.

20 Le moment où les Allemands sont entassés dans les geôles, c'est de la grande fiction. Les Wiener Philharmoniker jouent un très beau thème en mineur, pendant que Ucicky fait un travelling sur les prisonniers serrés, puis sur les visages. C'est du cinéma de fiction type, bien fait, efficace, le même qu'on verra ensuite et qui montrera les juifs dans les camps nazis : les expressions des visages, sur lesquels la lumière varie, en train de prier ou d'espérer, l'attente, le silence et les chuchotements, un vieux qui parle de la peur, le petit garçon qui dit qu'il n'en peut plus de rester debout tellement tous sont entassés les uns contre les autres, des personnes qui craquent et se mettent à hurler, etc. Tout y est, intensifié par la remarquable gestion du temps, l'alternance de lents travellings et de plans fixes ${ }^{11}$.

21 On comprend du coup les raisons pour lesquels Claude Lanzmann, l'auteur de Shoah, critique la représentation de l'extermination des juifs donnée par la mini-série américaine Holocaust ou La Liste de Schindler. C'est que, par opposition au film de Lanzmann et à son travail pour rendre surprésente la Shoah qui, pourtant, n'est jamais 
figurée, montrer équivaut nécessairement à tomber dans l'imposture, puisque c'est précisément utiliser les mêmes codes, les mêmes stratégies que ceux avec lesquels les nazis montraient l'extermination des Allemands par les Polonais, c'est-à-dire en définitive les stratégies avec lesquelles on peut justifier n'importe quoi. Par là, c'est la Shoah qui devient n'importe quoi. Pour quelque chose de cet ordre, la Shoah, il y a donc une perversion constitutive de la fiction qui fait qu'il est impossible de la constituer en objet d'une quelconque figuration. Il n'y a, structurellement, aucune différence entre les juifs qui entrent dans les chambres à gaz et les Allemands qu'on entasse, à la fin de Heimkehr, dans des caves afin de les exterminer.

\section{BIBLIOGRAPHIE}

AscheId Antje (2003), Hitler's Heroines. Stardom and Womanhood in Nazi Cinema, Philadelphie :

Temple University Press.

AYÇOBERRY Pierre (1998), La société allemande sous le III Reich. 1933-1945, Paris : Seuil.

BRocKMANN Stephen (2010), A Critical History of German Film, Rochester : Camden House.

BÜTTNER Elisabeth (2007), « Du rapport entre l'histoire du cinéma autrichien et l'histoire », Austriac, 64 (Le Cinéma autrichien).

CAPURRo Rafael \& GRIMM Petra (dir.) (2004), Krieg und Medien, Stuttgart : Steiner Verlag.

DuFouR Éric (2006), Le Cinéma d'horreur et ses figures, Paris : PUF.

Dufour Éric (2014), Le Mal dans le cinéma allemand, Paris : Armand Colin.

FREI Norbert (1994), L'État hitlérien et la société allemande, trad. fr. J. Etoré, Paris : Seuil.

Fox Jo (2000), Filming Women in the Third Reich, Oxford : Berg.

Fox Jo (2007), Film Propaganda in Britain and Nazi Germany, Oxford : Berg Publishers.

HARDOUIN-FUGIER Élisabeth (2002), « La protection de l'animal sous le nazisme », Luc Ferry ou le rétablissement de l'ordre (129-151), Lyon : Éditions Tahin Party.

HELM Laura (2006), Der Film im NS-Regime, Munich : Grin Verlag.

HoRKHEIMER M. (1978), « La dernière attaque contre la métaphysique », Théorie critique, Paris, Payot.

KLEMPERER Victor (1996), La Langue du III Reich, Paris : Albin Michel [Leipzig, 1975].

KRAH Hans (dir.) (2000), Geschichte(n), NS-Film - NS Spuren heute, Kiel : Ludwig.

RENTSCHLER Eric (2002), The Ministry of Illusion. Nazi Cinema and Its Afterlife, Harvard University Press.

SANDER Anneliese Ursula (1944), Jugend et Film, Berlin : Gerold Verlag.

TRIMMEL Gerald (1998), Heimkehr: Strategien eines NS Films, Vienne : Werner Eichbauer. 
WelCH David (2006), Propaganda and the German Cinema, 1933-1945, Londres : I. B. Tauris.

\section{NOTES}

1. G. Trimmel, «Der nationalsozialistische Spielfilm „Heimkehr“ und die Umsetzung der nationalsozialistischen Vernichtungspolitik in Polen», en ligne sur <www.donau-uni.ac.at/ imperia/md/images/department/kunstbildwissenschaft/

der_nationalsozialistische__spielfilm_heimkehr.pdf> ; "Der nationalsozialistische Spielfilm " Heimkehr". Strategien der Manipulation und Propaganda », en ligne sur <www.donau-uni.ac.at/ imperia/md/content/studium/kultur/film/heimkehr.pdf>.

2. Ibid. ; voir aussi Welch (2006 : 112) qui affirme la même chose sans donner plus d'arguments.

3. Sur le lien particulier entre les juifs et la Pologne dans l'idéologie nazie, voir, dans Der ewige Jude, la carte du monde avec la propagation des juifs dans le monde et la comparaison avec les rats : voir l'exposé de Claire Asgulo-Rallo, « Le Juif éternel (Der ewige Jude) de Fritz Hippler (1940) ».

4. Voir sur ce point Dufour (2006).

5. Voir G. Trimmel, «Der nationalsozialistische Spielfilm „Heimkehr“. Strategien der Manipulation und Propaganda », art. cité.

6. Pour le rôle de la musique vocale, on se reportera à l'exposé de Rémi Sanvoisin : « Ewiger Wald (1936, Hans Springer et Rolf von Sonjevski-Jawrowski). Art total et instrumentalisation de la musique dans le cinéma nazi ». Soulignons que, dans notre scène, Heimkehr met en évidence la fonction sociale de la musique (elle nous réunit et fonde, au sein de cet acte par lequel nous chantons une chanson sur le Heimat, la fusion de l'individu dans le tout) : c'est comme une autoréférence, par quoi Heimkehr, comme film et donc œuvre d'art au même titre que n'importe quelle chanson, manifeste son véritable statut, c'est-à-dire sa subordination à une fonction sociale très précise qu'est le fait de faire valoir la grande Allemagne. C'est en ce sens qu'il n'y a pas de divertissement apolitique dans le cinéma nazi.

7. Horkheimer écrit à propos du fascisme italien: «La prétention de Mussolini déclarant "provenir immédiatement des orientations les plus actuelles de l'esprit européen et spécialement des courants relativistes de la philosophie", trouve sa confirmation dans le fait que le mouvement qu'il conduisait ne s'était fixé aucun programme, se dénommant selon la situation aristocratique ou démocratique, révolutionnaire ou réactionnaire, prolétarien ou antiprolétarien, pacifiste ou antipacifiste. » $(1978: 243)$

8. Cette perspective sur l'idéologie nazie a en outre un avantage: elle interdit, contre une certaine lecture contemporaine du cinéma nazi dit "de divertissement », de prétendre qu'on trouverait dans celui-ci une certaine forme de résistance et de contestation de «l'idéologie nazie ». Voir Dufour (2014).

9. G. Trimmel, «Der nationalsozialistische Spielfilm „Heimkehr“. Strategien der Manipulation und Propaganda », art. cité.

10. Sur Ucicky, voir les quelques lignes dans Büttner (2007: 16).

11. C'est, par l'investissement affectif causé par l'immersion du spectateur au sein de la diégèse grâce au talent du scénariste et du réalisateur, une quasi expérience que fait ce spectateur des terribles atrocités auxquelles sont soumis les Allemands - complémentarité de la vue objective, où le spectateur n'est pas impliqué, des documentaires qui traitent le même thème dans le même sens (Feuertaufe, Feldzug in Polen) : voir Brockmann (2010:46). 


\section{RÉSUMÉS}

Cette contribution traite du film de Gustav Ucicky, Heimkehr (1941). Ce film appartient à la propagande nazie par laquelle celle-ci cherche à justifier l'occupation de la Pologne. À l'occasion de l'analyse de ce film, je montre qu'il n'y a pas chez les nazis d'idéologie positive. Ils utilisent tout ce qui est utile dans une situation particulière pour faire valoir quelque chose (ici l'occupation de la Pologne). Ainsi mettent-ils en avant dans ce film (comme dans le documentaire Maladie héréditaire) la valeur de l'humanisme. En outre, l'analyse montre comment Ucicky est un réalisateur doué, qui utilise toutes les forces de la langue cinématographique pour montrer l'exclusion des Allemands et pour toucher et influencer le spectateur. Ce sont les mêmes moyens filmiques qui seront utilisés ensuite, dans les films d'après-guerre (comme Holocaust ou La Liste de Schindler), par les films sur l'extermination des juifs.

Gustav Ucickys Film Heimkehr (1941) gehört zu den Propagandafilmen, durch welche die Nazis die Besetzung Polens zu rechtfertigen suchten. Anlässlich der Untersuchung dieses Filmes zeige ich, dass es bei den Nazis keine positive Ideologie gibt. Sie benutzen immer alles, was ihren politischen Zielen (hier der Besetzung Polen) dienen kann. So geben sie in diesem Film (wie im Kulturfilm Erbkrank) dem Wert des Humanismus den Vorrang. Außerdem zeigt die Analyse, dass Ucicky ein begabter Regisseur ist, der alle filmischen Stilmittel benutzt, um die Unterdrückung der Deutschen und den Sadismus der Polen zu zeigen und somit die Zuschauer zu rühren. Ausgerechnet diese filmischen Stilmittel greifen Nachkriegsfilme wie Holocaust oder Schindlers Liste auf, um mit den Opfern der nationalsozialistischen Massenverbrechen Mitleid zu erwecken.

\section{INDEX}

Schlüsselwörter : Heimkehr, Film im Dritten Reich, NS-Weltanschauung, Propagandafilm, Humanismus, Menschenwürde, Tiere, Frau, Polen, polnisches Volk, Deutschland, deutsches Volk Mots-clés : retour, cinéma nazi, idéologie nazie, film de propagande, humanisme, dignité humaine, animaux, femme, Pologne, Polonais, Allemagne, Allemand

\section{AUTEUR}

\section{ÉRIC DUFOUR}

Université Paris 7 